\title{
Self-reported disability and handicap after hearing-aid fitting and benefit of hearing aids: comparison of fitting procedures, degree of hearing loss, experience with hearing aids and uni- and bilateral fittings
}

\author{
Mick Metselaar • Bert Maat • Pieta Krijnen • \\ Hans Verschuure · Wouter A. Dreschler • \\ Louw Feenstra
}

Received: 19 June 2008 / Accepted: 13 October 2008 / Published online: 13 November 2008

(c) The Author(s) 2008. This article is published with open access at Springerlink.com

\begin{abstract}
Self-reported outcome on hearing disability and handicap as well as overall health-related quality of life were measured after hearing-aid fitting in a large-scale clinical population. Fitting was performed according to two different procedures in a double-blind study design. We used a comparative procedure based on optimizing speech intelligibility scores and a strictly implemented fitting formula. Hearing disability and handicap were assessed with the hearing handicap and disability inventory and benefit of hearing aids with the abbreviated profile of hearing aid benefit. Effects on health-related quality of life and depression were assessed with the EuroQol-5D questionnaire and the geriatric depression scale. We found that hearing-aid fitting according to either procedure had a significantly positive effect on disability and handicap associated with hearing loss. This effect lasted for several months. Only the effect on disability persisted after 1-year of follow-up. Selfreported benefit from hearing aids was comparable for both fitting procedures. Unaided hearing disability was more pronounced in groups of participants with greater hearing loss, while the benefit of hearing aids was independent from the degree of hearing impairment. First-time hearing aid users reported greater benefit from their hearing aids. The
\end{abstract}

M. Metselaar $(\bowtie) \cdot$ H. Verschuure $\cdot$ L. Feenstra

Department of Otolaryngology/Audiology,

Erasmus Medical Center, Dr. Molewaterplein 40,

3015 GD Rotterdam, The Netherlands

e-mail: r.m.metselaar@erasmusmc.nl

B. Maat · W. A. Dreschler

Department of Clinical and Experimental Audiology,

Academic Medical Center, Amsterdam, The Netherlands

P. Krijnen

Department of Public Health,

Erasmus Medical Center, Rotterdam, The Netherlands added value from a bilateral hearing-aid fitting was not significant. Overall health-related quality of life and incidence of depression did not alter after hearing-aid fitting.

Keywords Fitting hearing aids $\cdot$ Hearing impairment . Hearing disability · Self-report · Quality of life

\section{Introduction}

Hearing impairment has a negative effect on the healthrelated quality of life in elderly persons, due to communication difficulties [6]. Effects on social, emotional, communicative, and cognitive functioning can be partly compensated with hearing aids [13]. Although the whole process of auditory rehabilitation focuses on many more aspects such as the learning of communication strategies and adaptation to the acoustical environment, hearing-aid fitting is one of the first essential steps.

The consequences of hearing impairment can be investigated in the domains of disability and handicap according to the conceptual framework proposed by the WHO in 1980 [22]. Since 2001, the WHO has replaced these terms by 'activity limitation' and 'participation restriction' in their International Classification of Functioning, Disability and Health [23].

From the literature, little is known about the extent to which hearing-aid fitting procedures succeed in alleviating the consequences of activity limitation and restriction of participation, suffered by a hearing-impaired individual or by a group of hearing-impaired subjects fitted with hearing aids. Reports from comparisons of different types of fitting procedures in large-scale clinical populations are scarce.

We compared a comparative hearing aid selection and fitting approach with a strictly implemented prescriptive 
method in a double-blind randomized clinical trial [11]. A comparative procedure principally approximates the primary criterion (e.g. speech intelligibility, sound quality) chosen as close as possible. This offers direct clinical evaluation with the hearing aid in place. However, a comparative way of fitting could be expected to be more time-consuming and to be dependent on the knowledge and experience of the hearing aid fitter. A prescriptive method is based on a fitting formula that usually has been derived from physical data and clinical research. A fitting formula can easily be automated and offers a quick and reproducible method for the initial hearing aid selection. While the number of prescriptive formulae is gradually increasing, and the comparative fitting approach seems to steadily lose popularity, little is known about the effects of these types of fitting procedures on self-reported hearing disability and handicap and on overall health-related quality of life.

We performed this study to answer the following questions:

- Does a group of hearing-impaired patients report differences in hearing-specific and in general health-related quality of life after hearing-aid fitting according to a comparative or a prescriptive fitting procedure?

- Which characteristics of hearing-impaired populations are related to changes in self-reported hearing-specific and general health-related quality of life after hearing-aid fitting?

- Are changes in self-reported hearing-specific and general health-related quality of life preserved during 1-year follow-up?

- To what extent are hearing-specific and general healthrelated quality of life measures able to assess the effects of rehabilitation with hearing aids?

\section{Material and methods}

Population

All patients included in this study visited the department of Clinical and Experimental Audiology in the Academic Medical Center in Amsterdam or the Audiology Department of the Erasmus Medical Center in Rotterdam, the Netherlands, because of hearing-impairment over a period of at least 3 years.

The main criterion for auditory rehabilitation with hearing aids was an average pure-tone audiometric threshold of more than $35 \mathrm{~dB}$ at the better ear (insurance company criterion for partly reimbursing hearing aid expenses in the Netherlands). We included purely sensorineural hearing losses and mixed losses with a dominant sensorineural component. First-time candidates as well as experienced hearing aid users were included after having obtained informed consent.

\section{Exclusion criteria were}

- A maximum speech score in quiet of less than $50 \%$ on the better ear

- A retrocochlear hearing loss

- Meniere's disease (active phase)

- (Severe) tinnitus

- Significant co-morbidity

- Not being capable of answering the questionnaires or not being able to understand and speak the Dutch language to a sufficient amount.

The possibility of withdrawal from participation at any moment during the study was guaranteed.

Study design

A double-blind randomized study design was followed that has been described in detail previously [12]. Stratification was performed according to maximum (unaided) speech intelligibility at the better ear. Three strata of speech intelligibility were distinguished (50-74, 75-89, 90-100\%).

The aim of the comparative approach was to improve speech perception as much as possible, at least to the maximum speech intelligibility found in the (unaided) speech audiogram. For each fitting, a number of possibly suitable hearing aids were selected by the hearing aid fitter. This selection was based on both the hearing thresholds of the patient and the experience of the fitter. Free-field speech intelligibility in the quiet was compared with each of the selected hearing aids in situ and served as the primary selection criterion. A second criterion was used, based on sound quality judgements by the patient. This procedure has been described in detail by Verschuure [21].

The prescriptive procedure applied was based on the NAL-RP formula $[1,2]$ with the modification for profound hearing losses [3]. The formula has been designed to prescribe linear amplification for mild to profound sensorineural hearing losses. Strict implementation of the prescriptive method was made possible by use of a computerized selection and fitting program that was written exclusively for this study.

Once included in the study, each participant was initially fitted according to the comparative as well as the prescriptive procedure in an arbitrary sequence. This was done by different hearing-aid fitters who were not informed about each other's results, except for the type of hearing aid prescribed [behind-the-ear (BTE) or in-the-ear (ITE) hearing aid] and the ear(s) to be fitted. Unilateral as well as bilateral fittings were performed. These choices were determined by the first hearing-aid fitter and were kept the same for both prescriptions. 
We have chosen to fit hearing aids that were adjusted to provide linear amplification. This was prescribed as much as possible in order to provide us with a set of precisely predictable output characteristics, enabling accurate fitting according to the NAL-RP formula. Moreover, it facilitated random swapping within the range of possibly suitable hearing aids, allowing a uniform comparison and selection. No digital and WDRC-compression hearing aids were used as clear fitting procedures were lacking at the time of inclusion of the participants in our study.

Both selection procedures resulted in a prescription for a specific brand and model of a hearing aid with an exact specification of the settings (gain, tone settings, and maximum output) as well as the type of earmold. One out of these two prescriptions was randomly given to the patient. Hearing aids were actually provided by the hearing-aid acoustician one to two weeks after randomisation. This period was required to get the hearing aids delivered by the manufacturer and the ear mold produced. Hearing aids fitted according to the prescriptive procedure were adjusted as closely as possible to the calculated target, which was confirmed by insertion gain measurements. Hearing aids provided in the other group were adjusted according to the settings that were finally found during the initial evaluation process.

Each patient was given the hearing aid(s) on trial for a 12-week period of acclimatization and experience. In case of a comparative fitting, hearing aids were examined once halfway this period and further adjusted if necessary. Aided speech intelligibility was used as the main criterion. Just for keeping the fitting procedure hidden/blinded to all clients, the prescriptive fitting group also visited the Audiological Center. This 'dummy' visit was used for the completion of some of the questionnaires (Table 1).

Table 1 Moments of completion of all questionnaires used in this study

\begin{tabular}{ll}
\hline Moment of completion & Questionnaire \\
\hline$t=0$ 'randomisation' & HHDI \\
& GDS \\
& EQ-5D \\
$t=2$ weeks & APHAB (baseline profile) \\
$t=6$ weeks & APHAB \\
$\mathrm{t}=12$ weeks & HHDI \\
& EQ-5D \\
$t=6$ months & GDS \\
$t=12$ months & APHAB \\
& HHDI \\
& GDS \\
& EQ-5D
\end{tabular}

See text for the explanation of abbreviations
At the end of his or her 12-week evaluation-period, the study protocol of each patient was closed. The result of the fitting was assessed for its success. This was done by an independent audiologist (not the investigator) who measured aided speech intelligibility in the quiet and in noise. In case of a satisfactory result, the opinion of the patient was asked for. When the patient was also satisfied with the result, the hearing-aid fitting was finalized. Analysis of the data in this study has been performed on data derived from successfully fitted patients only. When the patient was not satisfied after fitting according to the prescriptive method, he was offered a fitting according to the comparative procedure that was being regarded as the golden standard. Dissatisfaction with the comparative procedure could occur in case of a request for re-fitting with a specific kind of digital or WDRC-hearing aid or for example with an ITE instead of an initially chosen BTE-hearing aid. From this phase on, the blinding was ended.

\section{Questionnaires}

To assess the effects of hearing-aid fitting on the experienced hearing disability and handicap and on the general and psychological well-being of hearing-impaired subjects, a number of validated hearing-specific and overall healthrelated measures were chosen.

1. Hearing handicap and disability inventory (HHDI). This questionnaire measures the consequences of hearing impairment in the domains of disability and handicap, according to the conceptual framework proposed by the WHO [23]. Disability is measured by subscale 'performance', while three handicap subscales are used: 'emotional response', 'social withdrawal', and 'reactions of others'. The latter subscale consists of the subscales 'positive' and 'negative reactions of others'. All items were scored in four response categories (range 1-4). Higher scores represent more disability or handicap [18].

2. Abbreviated profile of hearing aid benefit (APHAB). This self-report questionnaire quantifies disability associated with hearing loss in a number of acoustically different daily life situations [4]. Benefit of hearing aids was computed by subtracting the results of performance with the hearing aid during fitting (6 weeks) and after 6 months' follow-up from performance without the hearing aid (or with the previous hearing aid for experienced users) that was measured 2 weeks after randomization. The items are clustered in four subscales: 'ease of communication' (EC), 'background noise' (BN), 'reverberation' (RV), and 'aversiveness of sounds' (AV). The Dutch translation of the original text was cross-translated into the English 
language to verify the quality of the translation. Some of the listening situations had been adapted to the Dutch environment. All items were scored on a visual analog scale. Higher scores are indicating more problems. As an addition to the APHAB, also the frequency of occurrence, importance of understanding speech, and proportion of time the hearing aid was used were investigated for each listening situation, as proposed by Gatehouse [7]. This information determines to what extent the hearing aid contributes to subjective auditory functioning of the client and served as a weighing factor for each question.

3. The geriatric depression scale (GDS) is a self-rating screening scale for depression in the elderly population [24]. This scale has been validated for subjects over 55 years of age. We used the short version of the GDS [15]. This scale contains 15 propositions that can be answered with "yes" or "no". Depression was diagnosed when more than 5 out of 15 items were scored positive.

4. The EuroQol-5-dimensions instrument (EQ-5D) is a generic self-report questionnaire consisting of two parts [17]. The first part records self-reported problems on each of five different dimensions: mobility, selfcare, usual activities, pain/discomfort, and anxiety/ depression. Each dimension is divided into three levels of severity corresponding to no problem, some problem, and extreme problem. Applying a weighing system [19], outcomes of this part can be presented as a single health index (EQ-5D $\left.\mathrm{D}_{\text {index }}\right)$. The second part records self-assessed rating of general health on a visual analogue scale $\left(\mathrm{EQ}-5 \mathrm{D}_{\text {vas }}\right)$. This scale ranges from 0 to 100 , representing worst to best imaginable health condition, respectively.

All questionnaires were self-administered and were completed at three different moments during the fitting process. Help was being offered when necessary in order to avoid unanswered questions as much as possible. The moments of completion of the questionnaires differed and are given in Table 1. However, the timing scheme was equal for both fitting procedures.

Because of the fairly large amount of questionnaires used in this study, we divided the moments of completion among the several visits necessary for hearing-aid fitting and evaluation. As a result of this, the first APHAB was completed 2 weeks after randomisation. Because the actual hearing aids were not really fitted to the clients before that time, we regarded this moment as 'baseline' as well. Although some patients had to be encouraged somewhat, most of them did not really object to the workload caused by completion of the questionnaires.
Statistical analysis

All data were analysed using SPSS software release 12.0.1 (SPSS Inc.). Differences between group averages were tested with the Student $t$ test. When distribution functions of the data showed clear deviations from normality, nonparametric testing was performed. We used Wilcoxon's test for paired comparisons and the Mann-Whitney $U$ test for unpaired comparisons.

We first analysed differences in subjective outcome between comparative and prescriptive fittings. Subsequently, analysis was done for the following subgroups:

- The three strata of maximum speech intelligibility.

- Experienced versus first-time hearing-aid users.

- Unilateral versus bilateral fittings.

We thought these latter subgroups to be especially of interest for the APHAB questionnaire concerning the items that were clustered in subgroups 'background noise', 'reverberation', and 'aversiveness'.

\section{Results}

Population

In total, 254 hearing-impaired patients (163 men, 91 women) were included in the study. Age ranged from 29 to 95 years with an average age of 71 years and SD of 13.5 years. Average pure-tone audiogram thresholds (averaged over 1,2 and $4 \mathrm{kHz}$ ) were $57.5 \mathrm{~dB}$ HL ranging from 30.6 to $102.5 \mathrm{~dB}$ HL. Speech reception threshold (SRT) varied from 11.4 to $94.6 \mathrm{~dB}$ with a mean of $53.2 \mathrm{~dB}$. Thirty-four patients were included in the lower stratum, while 79 and 141 patients were included in the middle and upper stratum, respectively. We included 113 (44.5\%) firsttime hearing-aid users, and $196(77.2 \%)$ patients were fitted bilaterally. After randomization, 119 (46.9\%) patients were fitted according to the comparative procedure. About half of the participants (50.8\%) were recruited in Amsterdam. In 184 patients (72.4\%), hearing-aid fitting was regarded successful according to the aforementioned criteria.

Hearing handicap and disability inventory (HHDI)

Comparable scores on all HHDI-subscales were found before hearing-aid fitting in the comparative and prescriptive subgroups (see Fig. 1). Significant improvements (that means: corresponding to lower scores) were measured in both subgroups directly after fitting in the disability-subscale 'performance' and in two of the three handicap-subscales, 'emotional response' and 'withdrawal' $(P<0.001$; Wilcoxon). In the subscales 'performance' and 'emotional 
Fig. 1 a-d Scores on HHDIsubscales and standard errors at the three moments of completion of the questionnaire in the study for comparative fittings (open symbols) and prescriptive fittings (solid symbols). Lower scores represent better results
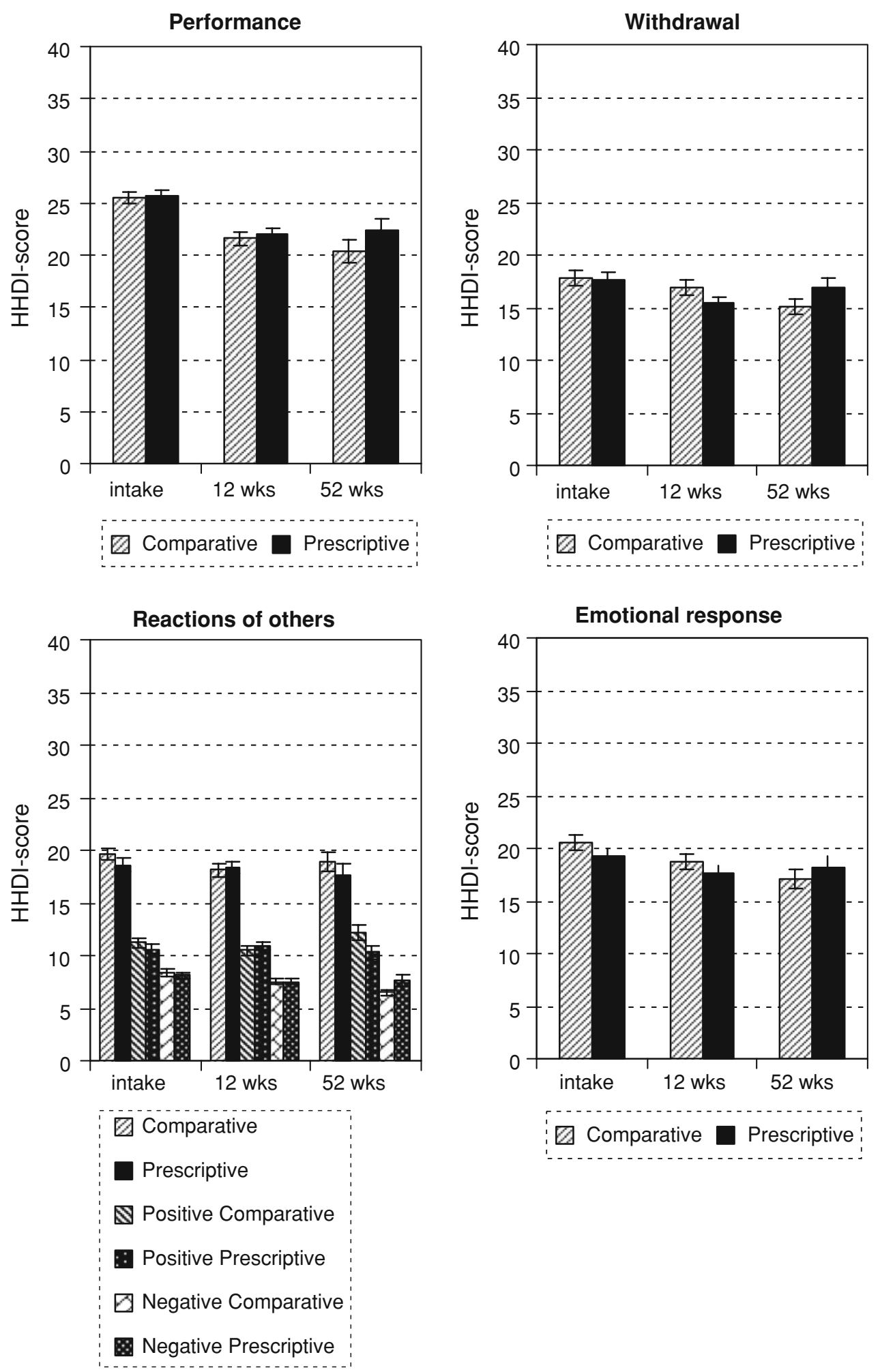

response' this effect was preserved during the 1-year follow-up period (Fig. 1a, d), while it disappeared in the subscale 'withdrawal' (Fig. 1b).

At the end of the fitting procedure (12 weeks), a significantly larger improvement on handicap-subscale 'withdrawal' was found for the prescriptive subgroup. This difference disappeared at 1-year follow-up. One year after fitting, differences between improvement were found to be significant in subscales 'performance' and 'negative reactions of others' $(P<0.05 ; \mathrm{M}-\mathrm{W} U$ test $)$ in favour of comparative procedure. 
Fig. 2 Average scores on the HHDI-questionnaire for the three moments of completion on subscale "performance" broken down after stratum of unaided speech discrimination (a) and "withdrawal" for inexperienced and experienced hearing aid users (b). Error bars show $\pm 1 \mathrm{SE}$

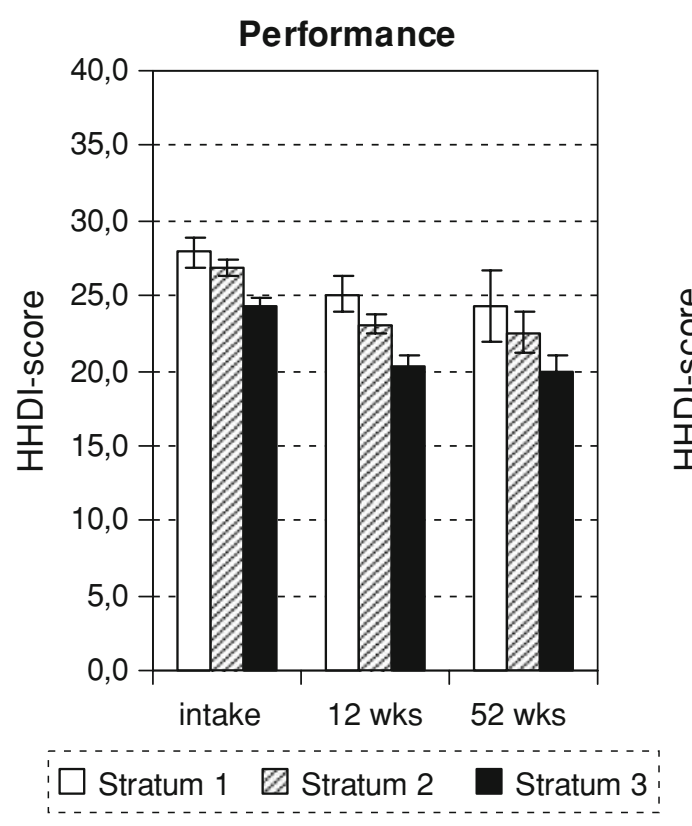

Withdrawal

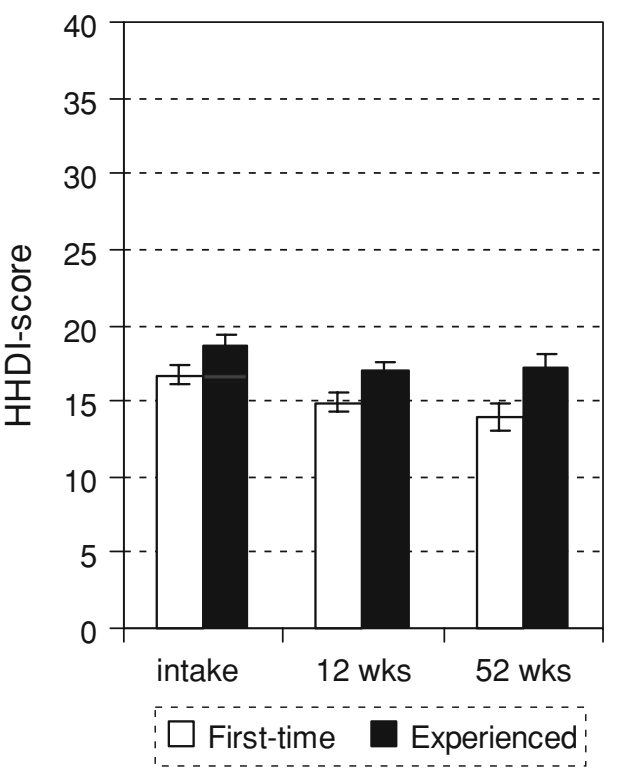

Next, we analysed the HHDI-scores for the three strata of maximum unaided speech discrimination (see Fig. 2). We found significant differences $(P<0.005)$ only in disability subscale 'performance' for the lower two strata (50-74 and 75-89\%) compared to the highest stratum (90-100\%). These differences were present before and after hearing-aid fitting and at 1-year followup and were better in the highest stratum (Fig. 2a). Hearing disability was not different between the lowest two strata.

Within the three strata of maximum unaided speech discrimination, no significant differences between the two fitting procedures were measured in any of the HHDI-subscales, except for the lowest stratum in subscale 'emotional response' directly after fitting (12 weeks) where a significant difference was measured in favour of the prescriptive procedure $(P<0.05 \mathrm{M}-\mathrm{W} U$ test $)$.

Clients that had not been wearing hearing aids earlier, scored significantly better only on handicap subscale 'withdrawal' compared to experienced users $(P<0.05)$ at all three moments of completion of the questionnaire (Fig. 2b). Scores on all other subscales were not significantly different for first-time and experienced hearing aid users.

We found no differences between the two fitting procedures amongst first-time and experienced hearingaid users on any of the HHDI-subscales, except for subscale 'performance' at 52 weeks. In this subscale first-time hearing-aid users scored significantly better when fitted according to the comparative procedure $(P<0.005)$.

No differences in any of the HHDI-subscales were measured between the two fitting procedures in the subgroups with unilateral and bilateral hearing-aid fittings.
Abbreviated profile of hearing-aid benefit (APHAB)

Significant benefit $(P<0.005$; Wilcoxon) in all subscales was measured during hearing-aid fitting (6 weeks), except in subscale aversiveness $(P>0.05$; Wilcoxon), see Fig. 3 . Benefit was preserved after 26 weeks follow-up except for the subscale aversiveness where again no difference was measured.

No significant differences in benefit measured at 6 and 26 weeks were found between the two fitting procedures in any subscale.

No significant differences in benefit between the three strata of maximum unaided speech recognition were found in any subscale on any moment of completion of this inventory.

Also, no differences in benefit between both fitting procedures were found in each of the three strata of maximum unaided speech discrimination.

First-time hearing-aid users reported significantly more benefit at 6 and 26 weeks compared to experienced users in subscales 'EC', 'BN', and 'RV'. In subscale 'AV' the benefit was negative: first-time users reported more problems compared to experienced users. Results are given in Table 2.

However, we found no differences in benefit in any of the APHAB-subscales for experienced and inexperienced users between both fitting procedures.

In the group with bilateral fittings, significantly $(P<0.05 ; \mathrm{M}-\mathrm{W} U$ test $)$ more benefit was reported only in acoustical circumstances with background noise (subscale 'BN') during hearing-aid fitting (6 weeks). This difference was not found after 6 months' follow-up. No differences were measured in the other subscales, either during fitting 
Fig. 3 Average APHAB-scores for the three moments of completion (before fitting, during fitting and at 6 months follow-up). Error bars show \pm 1 SD. Scores are shown for each APHAB subscale
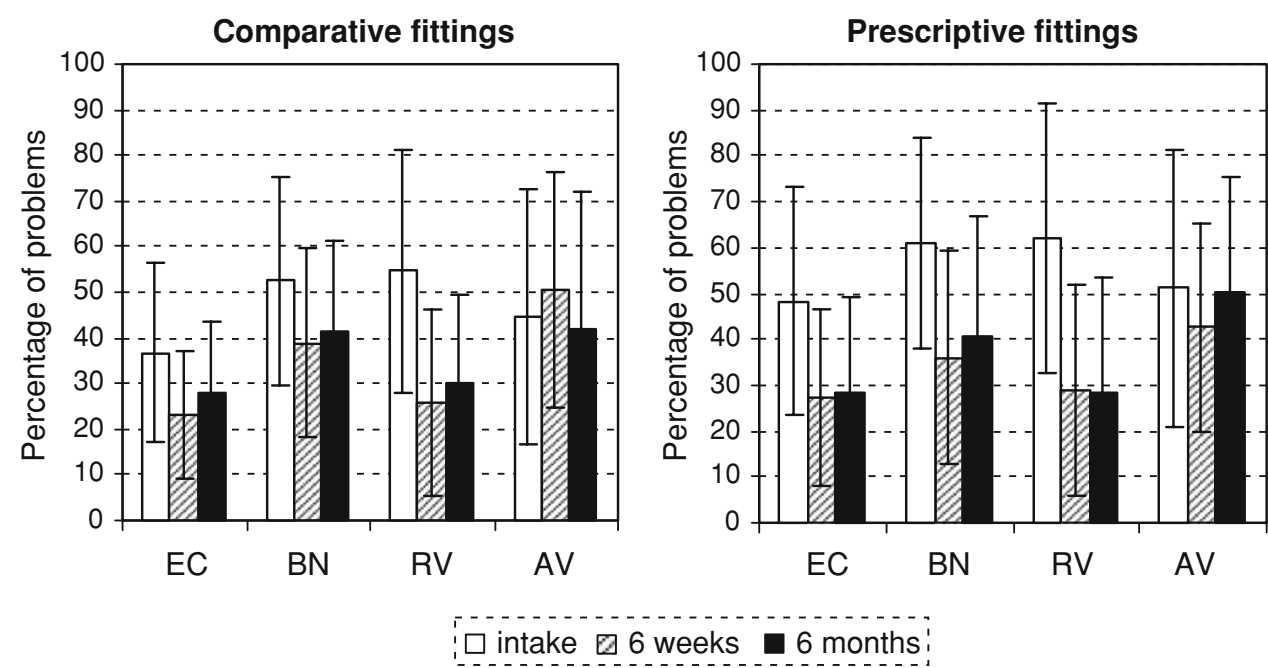

Table 2 Average benefit and standard deviations on all APHAB-subscales during (6 weeks) and after (26 weeks) hearing-aid fitting for first-time and experienced hearing aid users

\begin{tabular}{lllll}
\hline APHAB-subscale & \multicolumn{2}{l}{ First-time } & & \multicolumn{2}{l}{ Experienced } \\
\cline { 2 - 3 } & 6 weeks & 26 weeks & 6 weeks & $+15.8 \pm 23.6$ \\
\hline EC & $+27.2 \pm 26.1^{*}$ & $+25.8 \pm 29.9 * *$ & $+11.3 \pm 25.7$ & $+13.4 \pm 24.7$ \\
BN & $+33.2 \pm 28.1^{*}$ & $+27.8 \pm 27.9 * *$ & $+10.9 \pm 26.7$ & $+20.0 \pm 27.0$ \\
RV & $+45.8 \pm 31.3^{*}$ & $+42.9 \pm 32.7 * *$ & $+15.4 \pm 26.9$ & $+6.6 \pm 21.9$ \\
AV & $-12.7 \pm 29.1^{*}$ & $-8.4 \pm 37.8^{* *}$ & $+9.4 \pm 27.0$ & + \\
\hline
\end{tabular}

Benefit is indicated by positive numbers

* Significant $(P<0.005 ; \mathrm{M}-\mathrm{W} U$ test $)$ compared to experienced ha-users

** Significant $(P \leq 0.05)$ compared to experienced ha-users

Table 3 Average benefit and standard deviations on all APHAB-subscales after hearing-aid fitting (relative to the pre-fitting results) during fitting (6 weeks) and after long-term follow-up (26 weeks)

\begin{tabular}{lllll}
\hline APHAB- subscale & \multicolumn{2}{l}{ Unilateral } & & \multicolumn{2}{l}{ Bilateral } \\
\cline { 2 - 3 } & 6 weeks & 26 weeks & 6 weeks & 26 weeks \\
\hline EC & $+14.2 \pm 22.9$ & $+20.3 \pm 21.3$ & $+19.5 \pm 28.1$ & $+19.7 \pm 27.5$ \\
BN & $+10.9 \pm 25.2$ & $+14.3 \pm 26.0$ & $+23.7 \pm 30.0 *$ & $+19.7 \pm 27.0$ \\
RV & $+22.8 \pm 26.8$ & $+24.9 \pm 33.2$ & $+32.2 \pm 34.1$ & $+29.6 \pm 31.3$ \\
AV & $-0.8 \pm 25.7$ & $-13.6 \pm 26.0$ & $-0.4 \pm 31.1$ & $+2.3 \pm 30.6$ \\
\hline
\end{tabular}

Benefit is indicated by positive numbers

* Significant differences $(P<0.05 ; \mathrm{M}-\mathrm{W} U$ test $)$ are indicated compared to unilateral fittings

or after follow-up. No differences were measured between the two fitting procedures for subgroups with unilateral and bilateral hearing-aid fittings. Results are given in Table 3.

\section{Geriatric depression scale (GDS)}

The short (15-item) version of the GDS was completed at the baseline-time $(t=0)$, at the end of the hearing-aid fitting (12 weeks later), and 1-year after fitting. At baseline, 8.8\% of the study-population of over 55 years of age met the criteria of depression according to the GDS (average score 2.05; SD 2.44), which seems to be somewhat lower than compared to a random American population [8]. No clear correlations were found between GDS-score and age (Pearson correlation $-0.036 ; P>0.5)$ and between GDS-score and degree of hearing loss as represented by maximum unaided speech intelligibility (Pearson correlation 0.025; $P>0.5$ ). Average GDS-scores and percentages of depression remained stable directly after and 1 year after hearingaid fitting (1.57; 6.2 and $2.32 ; 8.3 \%$ respectively). No 
Fig. 4 Average GDS-scores during the three moments of completion (before and directly after hearing-aid fitting and after 1 year of follow-up). Error bars show \pm 1 SD. Difference between first-time users and experienced users is significant after 52 weeks
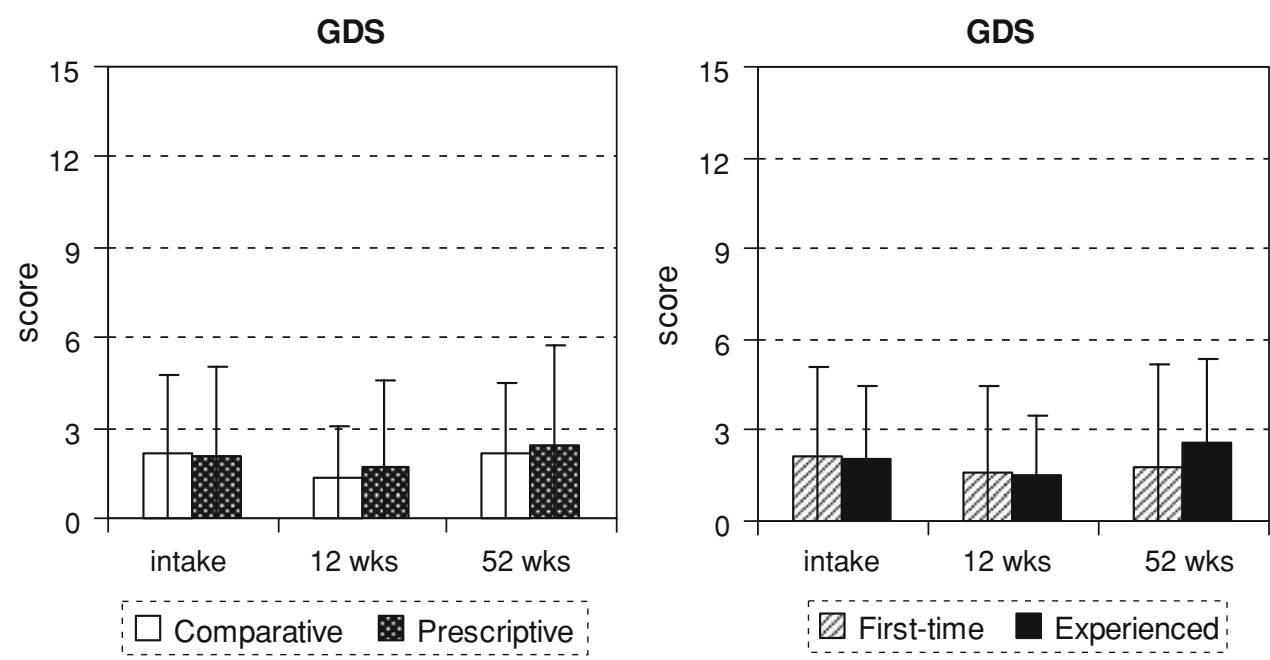

$\square$ First-time

Experienced:
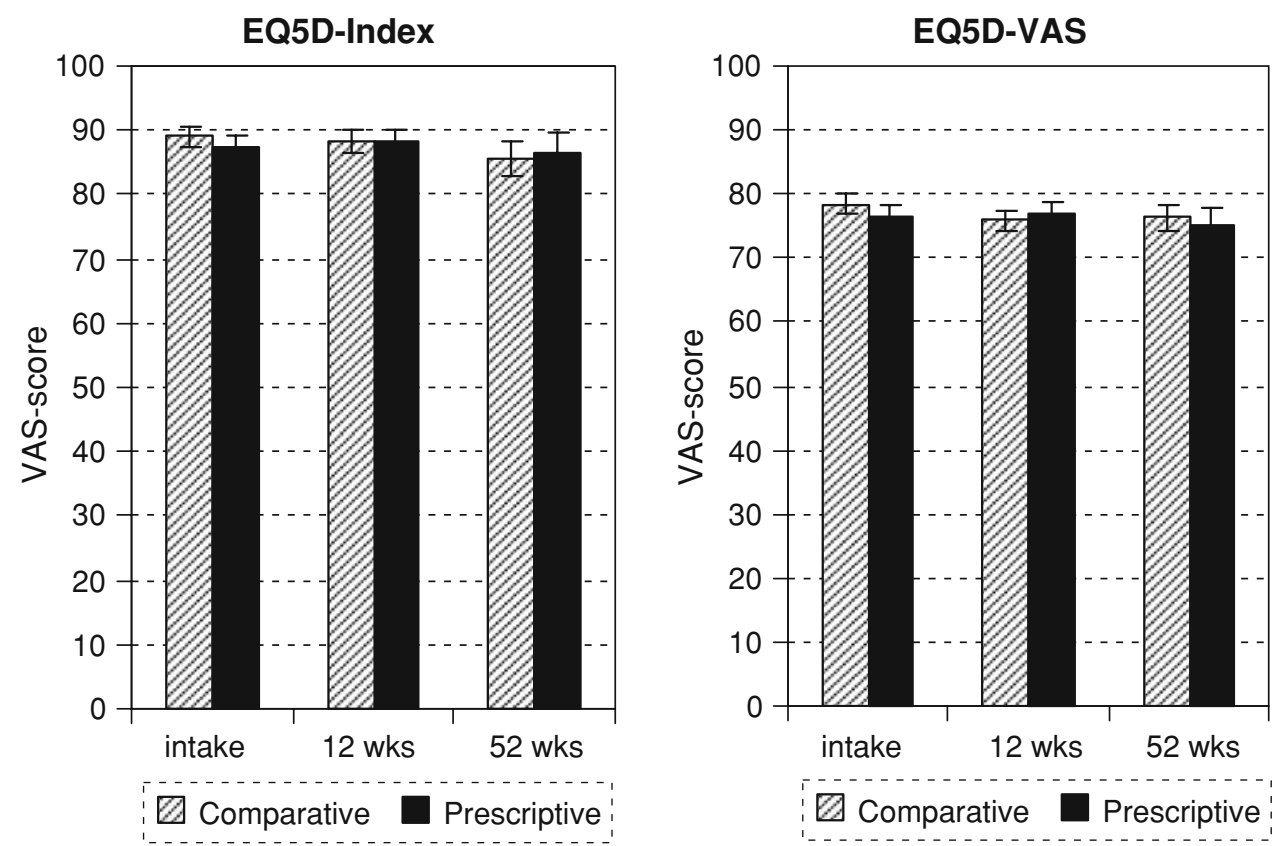

Fig. 5 a, b Scores on EuroQol health index and VAS at the three moments of completion for the complete population. Error bars represent standard errors

differences were found between the comparative and prescriptive subgroups (Fig. 4a). No significant differences were found between the three strata of maximum unaided speech discrimination. Experienced hearing aid users reported significantly higher GDS-scores $(P<0.05)$ compared to first-time users only after 1 year of follow-up (Fig. 4b).

We concluded that prevalence of depression according to the GDS in our population was relatively low and remained unchanged after fitting with hearing aids according to either procedure during a 1-year follow-up period.

\section{Euroqol-5D (EQ-5D)}

At baseline, EQ-5D $\mathrm{D}_{\text {index }}$ was 88.1. Correlation with age was significant (Pearson $-0.16 ; P<0.05$ ). No correlation with degree of hearing loss was found (Pearson $0.03 ; P>0.5$ ). Directly after fitting and after 1-year follow-up EQ-5D ${ }_{\text {index }}$ was 88.6 and 87.6, respectively. These numbers were not significantly different from baseline-situation $(P>0.05$; paired $t$ test). No differences between both fitting procedures were present (Fig. 5a).

The rating of general health on a visual-analogue scale (VAS) was initially $77.4(\mathrm{SD}=14.8)$ for the whole study population. Again, correlation with age was significant (Pearson $-0.16 ; P<0.05$ ). No correlation with degree of hearing loss was found (Pearson $-0.02 ; P>0.5$ ). Directly after fitting, the VAS-score was not significantly different from baseline (76.3, $P=0.7$; paired $t$ test). However, 1 year after fitting it was rated significantly lower $(75.6 ; P<0.05$; paired $t$ test). Results were similar for both fitting procedures (Fig. 5b). 
No significant differences were found between first-time and experienced hearing-aid users and between the three strata of maximum unaided speech discrimination.

We concluded that hearing-aid fitting did not alter selfreported general health according to the EuroQol-5D. A decrease in self-reported VAS-rating of general health over a 1-year follow-up period was observed in our study population. This was not related to the type of fitting procedure.

\section{Discussion}

This study is one of the few relatively large clinical studies to evaluate a comparative and a prescriptive fitting procedure in a randomized setting. We have chosen a linear fitting formula (NAL-RP) to fit hearing aids with linear amplification as much as possible in order to be able to predict the hearing-aid output most accurately.

We realize that, as a consequence of these choices, the results of the present study may not be extrapolated to modern digital nonlinear hearing aids. On the other hand, we would not have been able to perform such a comparison of selection and fitting procedures with the currently available modern hearing aids.

Summarizing the results of the present study, we found no consistent differences in self-reported hearing disability and handicap in favour of either fitting procedure. The supposed differences between the fitting procedures could therefore not clearly be established. A strictly implemented (computer-aided) prescriptive fitting procedure provides an equal amount of hearing-aid benefit and reduction of hearing disability and handicap to a comparative (adaptive) procedure, in which the hearing-aid can be fine-tuned according to the clients' suggestions after initial fitting. This finding has also been reported in a pilot study that investigated the effects of additional fine-tuning after hearing-aid fitting on self-reported benefit [5]. The authors found no significant differences between a group of firsttime hearing-aid users, able to adjust their aids after initial fitting and a group that was withheld from additional finetuning. It seems that the wearing of hearing-aid itself is primarily responsible for the benefit, rather than the specific procedure used to fit them.

We found that self-reported hearing disability according to the HHDI was dependent on the degree of hearing loss that has been classified in one of the three strata of maximum unaided speech intelligibility at the better ear. Disability was significantly more pronounced in the lower two strata compared to the highest stratum. Surprisingly, this difference was preserved directly after hearing-aid fitting and even after 1-year follow-up. Apparently, fitting with hearing aids did not wipe out the influence of the degree of the hearing loss on disability. This finding was in accor- dance with our data on the self-reported benefit of hearing aids (measured with the APHAB). We found that the extent of self-reported benefit of hearing-aid fitting was not dependent on the degree of hearing loss, and thus was comparable in the three strata. This finding was also reported in a study by Meister et al. [10], who evaluated the hearing-aid fittings of a large number of all different kinds of hearingimpaired listeners using a fairly extended inventory. One of their findings was that a more severely impaired hearing loss caused greater problems with hearing aids. In a study after the outcome of hearing-aid fitting, Stark and Hickson [16] also found a relationship between self-reported hearing disability and degree of hearing impairment. They measured disability with the Hearing Handicap Inventory for the Elderly (HHIE) [20] before and after hearing-aid fitting and found a significantly greater reduction in HHIE-scores for participants with a three-frequency average (3-FA) hearing loss of greater than $35 \mathrm{~dB}$, when compared to the reduction measured for those with a 3-FA hearing loss of less than $25 \mathrm{~dB}$. Although the study by Stark and Hickson also reports a dependence of hearing disability and hearing loss, their findings are clearly different from ours. These contradictory results might be explained by the fact that we did not include participants with a 3-FA hearing loss of less than $35 \mathrm{~dB}$ in our study. All of our participants met the criteria of the more severely hearing impaired group in the aforementioned study.

We found a rather limited self-reported surplus value of bilateral hearing-aid fittings compared to unilateral fittings. A temporarily positive effect for acoustical circumstances with background noise (APHAB subscale 'BN') was found during hearing-aid fitting that disappeared after follow-up of several months. No differences were found in the subscales 'Reverberation' and 'Aversiveness'. These data are in accordance with the literature. Noble and Gatehouse [14] used the speech, spatial and qualities of hearing scale (SSQ) for a study on self-reported hearing benefit for people fitted unilaterally and bilaterally. They found no benefit in various self-rated contexts of listening against relatively stationary competing noise. Benefit of two hearing aids over one was only reported in more challenging speech hearing contexts.

Finally, we found no effect of hearing-aid fitting on the quality of life measured with the generic EuroQol-5D (EQ5D) questionnaire. This finding is in accordance with the study by Joore et al. [9], who used the EQ-5D to measure the impact of hearing-aid fitting in a population of 80 inexperienced hearing-aid users. According to their results, the generic quality of life of hearing impaired people did not change directly after fitting with hearing aids. On the contrary, it declines with age, as we found after 1 year; both the EQ-5D index and the VAS to be lower than at the start of the study. The difference of the latter parameter was even 
significant. It is likely that the decrease can be explained by the progression of age during follow-up, as we found significant correlations with age for both the EQ-5D index and VAS.

We measured no significant changes on self-reported depression in elderly patients after hearing-aid fitting in this study. The association between hearing impairment and depression that was assessed with the GDS has been investigated in a population of 472 elderly individuals of which 106 were identified as hearing-impaired [12]. Although the authors found no significant relationship between depression and hearing loss, a relatively small but significant improvement in depression scores was measured after hearing-aid fitting.

Apparently, the generic questionnaires used in this study were not sensitive enough to detect changes in general health-related quality of life after hearing-aid fitting.

\section{Conclusions}

In this double-blind randomized clinical trial we have focused on self-reported outcome of hearing-aid fitting according to a comparative fitting procedure and a prescriptive method using a strictly implemented fitting formula (NAL-RP).

Our data were obtained from a large group of both experienced and first-time hearing-aid users with a varying degree of sensorineural hearing impairment. Hearing aids with linear amplification and analogue circuitry were prescribed. The conclusions listed below are thus primarily and possibly only relevant to this population, fitted with the kind of hearing aids that have been used in the study.

1. Hearing-aid fitting in general had a significantly positive effect on self-reported disability and handicap associated with hearing loss. This effect was measured after fitting according to either procedure investigated in this study. The effect on disability was preserved during a follow-up period of 1 year. Effects on handicap were less consistently durable.

2. We found no consistent difference in self-reported hearing disability and handicap between fitting according to a comparative procedure and a strictly implemented prescriptive method using a linear fitting formula.

3. Self-reported hearing disability was more pronounced in the lower two strata of maximum unaided speech discrimination compared to the highest stratum both before and directly after fitting and also after 1 year of follow-up.

4. Hearing-aid benefit was not dependent on the degree of hearing loss that was defined after maximum unaided speech discrimination at the better ear.
5. First-time hearing-aid users reported significantly less withdrawal (HHDI) than experienced users before fitting, directly after fitting and after 1-year follow-up. They also experience a larger degree of hearing-aid benefit compared to experienced users.

6. A bilateral hearing-aid fitting only temporarily results in more self-reported hearing-aid benefit in situations with background noise (APHAB).

7. No significant effects of hearing-aid fitting were measured on self-reported overall health-related quality of life (EQ-5D) and depression (GDS).

Acknowledgments This study has been conducted with financial support by the Dutch CVZ (College voor Zorgverzekeringen). The authors like to thank all the hearing-impaired patients that have participated in this study.

Open Access This article is distributed under the terms of the Creative Commons Attribution Noncommercial License which permits any noncommercial use, distribution, and reproduction in any medium, provided the original author(s) and source are credited.

\section{References}

1. Byrne D, Cotton S (1988) Evaluation of the National Acoustic Laboratories' new hearing aid selection procedure. J Speech Hear Res 31:178-186

2. Byrne D, Dillon H (1986) The National Acoustic Laboratories' (NAL) new procedure for selecting the gain and frequency response of a hearing aid. Ear Hear 7:257-265

3. Byrne D, Parkinson A, Newall P (1990) Hearing aid gain and frequency response requirements for the severely/profoundly hearing impaired. Ear Hear 11:40-49. doi:10.1097/00003446-19900200000009

4. Cox RM, Alexander GC (1995) The abbreviated profile of hearing aid benefit. Ear Hear 16:176-186. doi:10.1097/00003446199504000-00005

5. Cunningham DR, Williams KJ, Goldsmith LJ (2001) Effects of providing and withholding postfitting fine-tuning adjustments on outcome measures in novice hearing aid users: a pilot study. Am J Audiol 10:13-23. doi:10.1044/1059-0889(2001/001)

6. Dalton DS, Cruickshanks KJ, Klein BE, Klein R, Wiley TL, Nondahl DM (2003) The impact of hearing loss on quality of life in older adults. Gerontologist 43:661-668

7. Gatehouse S (1996) Outcome measures for hearing aid evaluation: properties and requirements in acclimatization research. Ear Hear 17:26S-28S

8. Gurland BJ (1976) The comparative frequency of depression in various adult age groups. J Gerontol 31:283-292

9. Joore MA, Brunenberg DE, Chenault MN, Antheunis LJ (2003) Societal effects of hearing aid fitting among the moderately hearing impaired. Int J Audiol 42:152-160

10. Metselaar RM, Maat B, Krijnen P, Verschuure J, Dreschler WA, Feenstra L (2008) Comparison of speech intelligibility in quiet and in noise after hearing aid fitting according to a purely prescriptive and a comparative fitting procedure. Eur Arch Otolaryngol 265:1113-1120 (epub ahead of print)

11. Meister H, Lausberg I, Kiessling J, von Wedel H, Walger M (2005) Detecting components of hearing aid fitting using a selfassessment-inventory. Eur Arch Otorhinolaryngol 262:580-586. doi:10.1007/s00405-004-0868-z 
12. Mulrow CD, Aguilar C, Endicott JE, Tuley MR, Velez R, Charlip WS, Rhodes MC, Hill JA, DeNino LA (1990) Quality-of-life changes and hearing impairment. Ann Intern Med 113:188-194

13. Mulrow CD, Aguilar C, Endicott JE, Velez R, Tuley MR, Charlip WS, Hill JA (1990) Association between hearing impairment and the quality of life of elderly individuals. J Am Geriatr Soc 38:45-50

14. Noble W, Gatehouse S (2006) Effects of bilateral versus unilateral hearing aid fitting on abilities measured by the speech, spatial and gualities of hearing scale (SSQ). Int J Audiol 45:172-181. doi:10.1080/14992020500376933

15. Sheikh JI, Yesavage YA (1986) GDS; recent evidence and development of a shorter version. In: Brink TL (ed) Clinical Gerontology. Haworth Press Inc., Binghamton

16. Stark P, Hickson L (2004) Outcomes of hearing aid fitting for older people with hearing impairment and their significant others. Int $\mathbf{J}$ Audiol 43:390-398. doi:10.1080/14992020400050050

17. The EuroQol Group (1990) EuroQol: a new facility for the measurement of health-related quality of life. Health Policy 16:199208. doi:10.1016/0168-8510(90)90421-9

18. van den Brink RHS, Wit HP, Kempen GIJM, van Heuvelen MJG (1996) Attitude and help-seeking for hearing impairment. Br J Audiol 30:313-324. doi:10.3109/03005369609076779
19. van Hout BA, McDonnell J (1992) Estimating a parametric relationship between health description and health valuation using the EuroQol Instrument. In: Björk S (ed) EuroQol Conference Proceedings. Institute for Health Economics, Lund (Sweden)

20. Ventry IM, Weinstein BE (1982) The hearing handicap inventory for the elderly: a new tool. Ear Hear 3:128-134. doi:10.1097/ 00003446-198205000-00006

21. Verschuure J (1994) Selektie van het hoortoestel. In: Kapteyn TS, Clemens A, Glazenburg BE, Joustra J (eds) Slechthorende en hoortoestel. NVA. De Leeuw Press, Rijnsburg

22. World Health Organization (1980) International classification of impairments, disabilities and handicaps. A manual of classification relating to the consequences of disease. World Health Organization, Geneva

23. World Health Organization (2001) International classification of functioning, disability and health (ICF). World Health Organization, Geneva

24. Yesavage JA, Brink TL, Rose TL, Lum O, Huang V, Adey M, Otto Leirer V (1983) Development and validation of a geriatric screening scale: a preliminary report. J Psych Res 17:37-49. doi:10.1016/0022-3956(82)90033-4 